\title{
ARTICLE
}

\section{Subnanometre enzyme mechanics probed by single-molecule force spectroscopy}

\author{
Benjamin Pelz', Gabriel Žoldák ${ }^{1}$ Fabian Zeller ${ }^{2}$, Martin Zacharias ${ }^{2}$ \& Matthias Rief ${ }^{1,3}$
}

Enzymes are molecular machines that bind substrates specifically, provide an adequate chemical environment for catalysis and exchange products rapidly, to ensure fast turnover rates. Direct information about the energetics that drive conformational changes is difficult to obtain. We used subnanometre single-molecule force spectroscopy to study the energetic drive of substrate-dependent lid closing in the enzyme adenylate kinase. Here we show that in the presence of the bisubstrate inhibitor diadenosine pentaphosphate (AP5A), closing and opening of both lids is cooperative and tightly coupled to inhibitor binding. Surprisingly, binding of the substrates ADP and ATP exhibits a much smaller energetic drive towards the fully closed state. Instead, we observe a new dominant energetic minimum with both lids half closed. Our results, combining experiment and molecular dynamics simulations, give detailed mechanical insights into how an enzyme can cope with the seemingly contradictory requirements of rapid substrate exchange and tight closing, to ensure efficient catalysis.

\footnotetext{
${ }^{1}$ Physik Department E22, Technische Universität München, James Franck Strasse 1, 85748 Garching, Germany. ${ }^{2}$ Physik Department T38, Technische Universität München, 85748 Garching, Germany. ${ }^{3}$ Munich Center for Integrated Protein Science, 81377 München, Germany. Correspondence and requests for materials should be addressed to M.R. (email: mrief@ph.tum.de).
} 
E:nzymes are machines that catalyse biochemical reactions in a highly dynamic process often involving large conformational changes. How conformational changes are coupled to catalytic turnover is still controversial ${ }^{1,2}$. Experimental techniques employed to answer those important questions are often limited and, although we have high-resolution crystal structures of selected states along the reaction pathways, capturing reaction dynamics and structure at the same time is difficult. As conformational changes are transitions in an energy landscape involving interatomic distance changes, enzymes may be viewed as molecular machines ${ }^{3}$. Single-molecule mechanical methods such as atomic force microscopy or optical tweezers have opened new ways for studying conformational transitions of molecular motors and in protein and nucleic acid folding ${ }^{4-9}$. In most enzymes, however, conformational motions associated with catalysis are generally very small $(<1 \mathrm{~nm})$, involving only few residues, and hence have so far not been directly amenable to those techniques. In this study, we have employed a high-resolution optical tweezers setup to study the mechanical coupling of substrate binding and lid closing in the enzyme adenylate kinase (AdK).

AdK catalyses the reversible conversion of ATP and AMP to two ADPs and thereby plays an important role for the energy balance of the cell. Adk consists of three domains. The ATP-and AMP-lid are responsible for binding of substrates and the CORE domain governs the overall stability of the enzyme ${ }^{10,11}$. During its catalytic cycle, AdK undergoes a large conformational change as both the ATP- and AMP-lid close over the CORE domain, thereby reducing unproductive active site fluctuations and minimizing non-productive hydrolysis ${ }^{12-14}$. Opening of AdK and subsequent product release have been suggested as the rate-limiting step for enzymatic turnover ${ }^{1,15,16}$. A variety of experiments and simulations have indicated that the enzyme samples closed-like states even in the absence of substrates ${ }^{16-19}$. Bisubstrate analogues consisting of two adenosine groups connected by a chain of phosphates, such as AP5A, have been proven strong inhibitors of $\mathrm{AdK}$ and bind with nanomolar affinity $^{20,21}$. AP5A is a multi-substrate inhibitor, which induces closing of the enzyme and keeps the lids firmly shut as has been shown by multiple crystal structures ${ }^{1,14}$. Therefore, the AP5A-bound state has served as a model for the fully closed (fc) state of AdK. Although crystal structures convey a picture of a tightly closed substrate-bound conformation, nuclear magnetic resonance (NMR) studies indicate much more dynamics ${ }^{1}$. In this study, we used single-molecule optical tweezers to directly measure the substrate-dependent forces that drive AdK into a closed conformation.

\section{Results}

Conformational dynamics in the presence of an inhibitor. We designed a mutant of a thermophilic variant of AdK from Aquifex aeolicus ${ }^{1}$, wherein we inserted cysteine residues at positions 42 and 144 that allowed us to bind DNA handles to the enzyme and then subject the molecule to mechanical loads in an optical trap (see Figs 1 and 2a). The residues were chosen such that the distance change between the two attachment points would be maximal $(c a .1 .7 \mathrm{~nm})$ on closing and opening of the lids as judged by the crystal structure (see Fig. 1). The variant showed wild-type activity for substrate turnover in bulk assays (see Methods). In a first set of experiments, we investigated the conformational fluctuations at constant trap positions (passive mode) in the presence of the inhibitor AP5A (see Fig. 2b). The distance between the two trap centres is held constant, imposing a constant average force bias on the fluctuating beads ${ }^{4,22}$. As only the distance between the two traps is held constant, the force acting on the protein-DNA system will vary and different forces will result in different DNA extensions that will add to the measured conformational change of the protein. To remove these linker effects, we performed a DNA elasticity correction (for details, see Methods). By changing the trap distance, the applied force bias can be varied and thus shifting the equilibrium of the conformational fluctuations of the protein.

In the presence of AP5A, we observed clear two-state transitions of AdK between an extended (lower level) and a contracted state (upper level) different by $c a .1 .6 \mathrm{~nm}$ in length. We identify the contracted conformation with the lid-closed conformation of AdK and the extended state with the lid-open conformation. This interpretation is supported by the finding that in the absence of an inhibitor, only the extended state is populated under load, while the contracted conformation is populated only in the presence of an inhibitor (see Supplementary Fig. 1). Our data directly show that closing and opening of both lids is cooperative, as the molecules always transition between the fully open and fc states. This interpretation is supported by a mutant where we attach our handles to only one lid (ATP lid) and the CORE domain (for details see Methods). We find conformational changes of only half the size $(c a .0 .9 \mathrm{~nm})$ albeit with identical kinetics (Supplementary Fig. 4).

The conformational transitions of AdK strictly depend on the concentration of inhibitor present (see Fig. 2c and Supplementary Fig. 5a). The closing rate rises proportionally to concentration over a large investigated range covering more than three orders of magnitude (Fig. 2c). In contrast, lid opening is independent of concentration (Fig. 2d and Supplementary Fig. 5b). The kinetics can be extrapolated to zero-force conditions (see asterisks in Fig. $2 c, d)$. The values we find for closing $\left(0.18 \mathrm{nM}^{-1} \mathrm{~s}^{-1}\right)$ and opening $\left(1 \mathrm{~s}^{-1}\right)$, as well as the associated equilibrium constant $(3 \mathrm{nM})$ almost exactly match published values for AP5A binding ${ }^{23}$ and affinity ${ }^{21}$ measured in bulk assays for Escherichia coli AdK. This agreement reveals a tight coupling of inhibitor binding and lid closing in the enzyme. Apparently, the complete gain in free energy on inhibitor binding is invested to keep the lids firmly shut.

Interestingly, the force dependence of the rates differs dramatically for lid closing as compared with opening. Although lid closing exhibits only a very weak dependence on force (shallow slopes in Fig. 2c), lid-opening rates couple much more strongly and change almost two orders of magnitude over the force range investigated (Fig. 2d). The independence of the closing rate on force provides direct evidence for an induced-fit mechanism of inhibitor binding. Induced-fit describes a binding mechanism where substrate binds to an open state and alters the
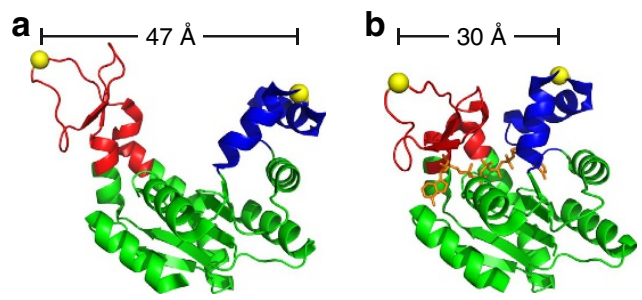

Figure 1 | Structure of AdK. The three-dimensional structure ${ }^{34}$ of the open (a) and closed (b) conformation of AdK from A. aeolicus (PDB ID Code $2 \mathrm{RH} 5$ and $2 \mathrm{RGX}$ ). The CORE domain is labelled in green, and ATP and AMP lid in red and blue, respectively. The closed conformation is shown in the presence of the bisubstrate inhibitor AP5A (orange). The yellow spheres indicate the position of the inserted cysteines between position 42 and 43 , and 144 and 145. The distance between the cysteines in the open and closed conformation is shown above. 
a

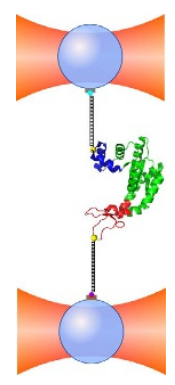

C

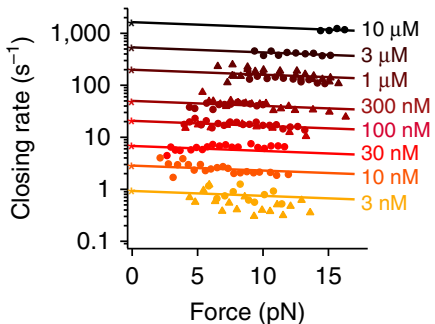

b

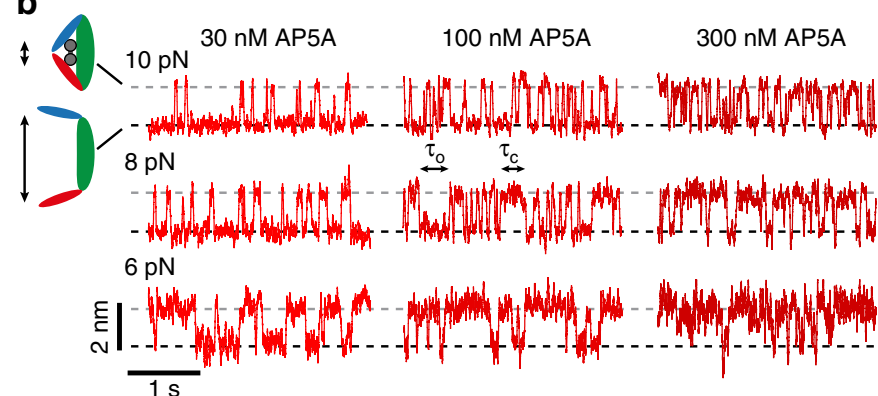

d

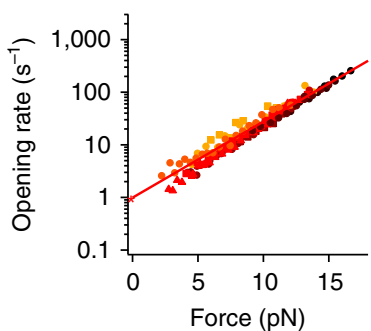

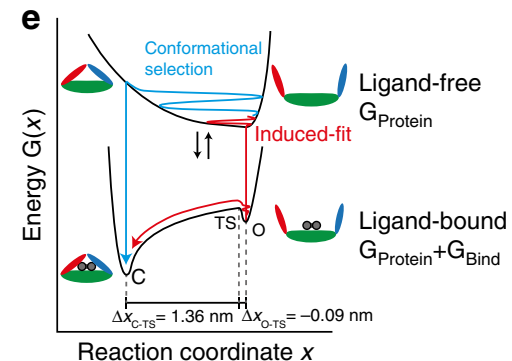

Figure 2 | Single-molecule force experiments of AdK by optical tweezers. (a) Optical trap assay (for details, see Methods). (b) Sample traces of the closing and opening fluctuations of AdK at different Mg-AP5A concentrations and force biases. The grey and black dashed lines indicate the position of the closed (contracted) and open (extended) state, respectively (see also Supplementary Fig. 2). (c) Closing rate as a function of force for different Mg-AP5A concentrations. Solid lines are extrapolations of the closing rates to zero force, which are indicated by the asterisks (for details, see Methods). (d) Opening rate as a function of force for different Mg-AP5A concentrations (colours as in c) (see also Supplementary Fig. 3). The solid line is a fit extrapolating the opening rate to zero force (asterisk). (e) Comparison of two binding and closing models for AdK and AP5A adapted from Okazaki and Takada ${ }^{45}$. Binding and unbinding of the inhibitor is represented as the jump between a ligand-free and ligand-bound energy landscape. Exemplary routes from open ligandfree to closed ligand-bound form are shown for the conformational selection (blue) and induced-fit model (red). For the ligand-bound energy landscape, distances from open and closed state to the transition state from optical trap experiments are shown.

energy landscape such that the protein is strongly driven into the closed state (see Fig. 2e red pathway). An alternative mechanism would involve a conformational selection where the protein fluctuates between open and closed conformations in the unbound state. Here, substrate recognizes the (rare) closed conformations in this ensemble, binds and locks the protein in this conformation (Fig. 2e blue pathway). As closing rates in our measurements are force independent, a conformational-selection mechanism can be ruled out, because force would reduce the probability for finding a closed conformation in the unbound state and hence also reduce binding and closing rates.

In a conventional view, force-dependent opening rates and force-independent closing rates indicate a transition state position close to the open state (see Fig. 2e bottom) further supporting the induced-fit mechanism. It is important to note that an induced-fit mechanism is well consistent with the unbound state being flexible and able to sample closed-like conformations as has been inferred from single-molecule fluorescence experiments and molecular dynamics (MD) simulations ${ }^{16-18}$. However, we can rule out that significant inhibitor binding occurs through those closed conformations, which is consistent with the crystal structure being so tightly closed that direct binding of inhibitor into the buried binding site appears unlikely ${ }^{14}$.

Nucleotide binding and conformational fluctuations. Under catalytic conditions with substrates, AdK has to be much more dynamic, as the enzyme must ensure rapid turnover. In a next set of experiments, we therefore investigated the lid-closing mechanics in the presence of the substrate molecules ATP and ADP. In contrast to AP5A, we could not detect any conformational kinetics in the presence of either of the substrate molecules (Supplementary Figs 6 and 7). Given the expected fast conformational fluctuations reported in NMR and in fluorescence experiments under saturating substrate conditions ${ }^{1,12,16}$, we suspected those fluctuations to be buried in the noise in our experiments. To assess the magnitude of the conformational transition with substrate, we therefore designed a competition experiment with fixed concentrations of AP5A and varying concentrations of substrate molecules (see Fig. 3a). This assay allows us to monitor the full conformational transition induced by AP $5 \mathrm{~A}$ and, at the same time, follow any changes induced by substrates.

With increasing ATP concentrations (Fig. 3a top to bottom), the open dwell times increase significantly, whereas the closed dwells remain unaffected. Apparently, ATP binds to the open state and blocks rebinding of AP5A. As an example, the open dwells increase their lifetime from $5 \mathrm{~ms}(0 \mu \mathrm{M}$ ATP, upper trace in Fig. 3a) to about $0.5 \mathrm{~s}$ at 3,000 $\mu \mathrm{M}$ (lowest trace in Fig. 3a). This means that during $99 \%$ of the open dwell time, ATP is bound to at least one binding site, therefore blocking the rebinding of AP5A from solution. This increase in dwell time provides a measure for the binding affinities of the various substrates to AdK (Fig. 3b and for details, see Methods). All affinities agree within error with published affinities from bulk experiments with no mechanical loads applied ${ }^{21}$, proving that our tethered enzyme has fully native substrate-binding properties. Further evidence that substrates bind directly to the enzyme even without AP5A present comes from experiments where we pulled AdK to forces where part of the structure unfolds (Supplementary Fig. 6). Constant velocity experiments in the presence of nucleotides (see Supplementary Fig. 6) suggest that the observed equilibrium transition is caused by the folding and unfolding of the ATP lid. This is supported by previous studies showing that the ATP and AMP lid of the mesophilic AdK from E. coli have a lower thermodynamic stability than the CORE domain with the ATP 
a
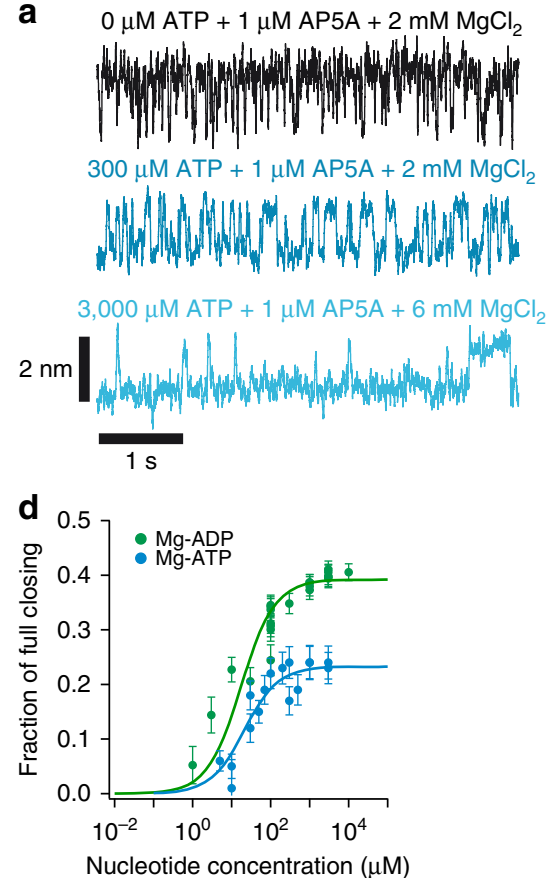

b

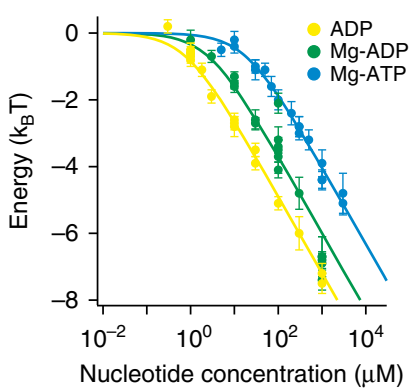

c

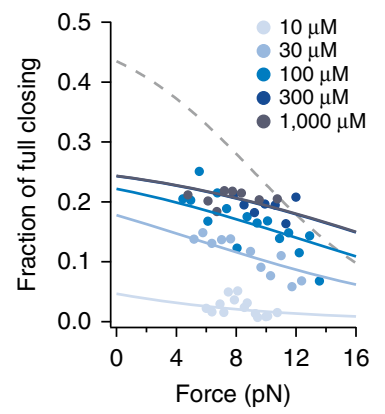

e

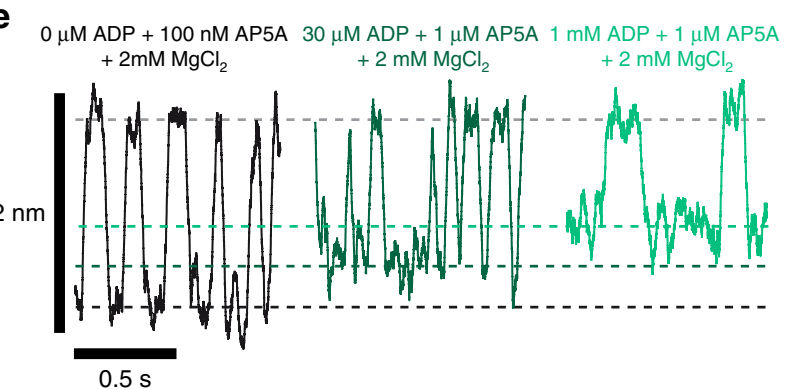

Figure 3 | Nucleotide-induced conformational changes of AdK. (a) Sample traces of AP5A-induced closing and opening in the single-molecule competition assay at $1 \mu \mathrm{M}$ AP5A with varying concentrations of ATP at a force of $10.5 \mathrm{pN}$. (b) Decrease in apparent binding free energy of AP5A in the single-molecule competition assay for the nucleotides ADP (yellow), Mg-ADP (green) and Mg-ATP (blue). Solid lines are fits of the binding model described in Methods (equation (2)) resulting in the following dissociation constants: $K_{D}^{A D P}=(0.8 \pm 0.1) \mu M, K_{D}^{M g-A D P}=(2 \pm 0.1) \mu M$ and $K_{D}^{M g-A T P}=(17 \pm 2) \mu M$. (c) $A T P$-induced fraction of full closing as a function of force for different ATP concentrations from the AP5A competition assay. Solid lines are fits to equation (7) yielding a size of the conformational change of $\Delta x=0.5 \mathrm{~nm}$. The dashed line shows the force-dependent closing expected if complete ATP lid closure is assumed. (d) Fraction of full closing at zero force induced by nucleotides Mg-ADP (green) and Mg-ATP (blue) as a function of nucleotide concentration extracted from the AP5A competition experiments. Solid lines are fits to the data assuming a binding model (equation (12)) with an affinity of the nucleotides to open and closed state of $\mathrm{AdK}$. The resulting dissociation constants are $K_{\text {close }}^{\mathrm{Mg}-\mathrm{ADP}}=(19 \pm 2) \mu \mathrm{M}, K_{\text {open }}^{\mathrm{Mg}-\mathrm{ADP}}=(290 \pm 80) \mu \mathrm{M}$ and $K_{\text {close }}^{\mathrm{Mg}-\mathrm{ATP}}=(25 \pm 5) \mu \mathrm{M}$ and $K_{\text {open }}^{\mathrm{Mg}-\mathrm{ATP}}=(134 \pm 60) \mu \mathrm{M}$. (e) ADP-dependent lid closing from AP5A competition experiments. The upper dashed line indicates the position of the fc AP5Abound state and the lower three lines indicate the position of the ADP-dependent AP5A-unbound state.

lid unfolding already at temperatures over $35^{\circ} \mathrm{C}$ (refs 11,24$)$. The higher conformational stability of the hyperthermophilic variant from A. aeolicus we use in our study explains why ATP lid melting is negligible at room temperature and needs substantial mechanical force to be induced. We observe that in the presence of substrates this equilibrium unfolding/folding transition is shifted to higher values demonstrating that substrate has bound and stabilized the protein structure. From these experiments we can independently extract dissociation constants from substrate binding, which lie in a very similar range as the ones obtained from the competition assay (for details, see Methods and Supplementary Fig. 8).

In contrast to AP5A (see above), the application of load apparently does not affect the binding of substrates to AdK as can be seen from the similarity of solution affinities with the ones we obtain at a pulling force of $10 \mathrm{pN}$. This provides evidence that substrate binding is not coupled to significant closing of the enzyme. Our competition assay also yields a direct comparison of the degree of lid closing between the substrate-bound and inhibitor-bound states. Even though our temporal resolution does not allow us to capture the individual conformational fluctuations induced by the rapid substrate binding/unbinding events, we can make a clear statement about the average degree of closing. Again, in the case of 3,000 $\mu \mathrm{M}$ ATP (see lower trace in Fig. 3a), the apparent closing distance between open and closed dwells induced by AP5A binding is almost identical to the one measured without ATP. This means that even though AdK carries ATP during $99 \%$ of the time in the open dwell, on average, the enzyme is nearly open. If ATP binding induced full closing of the ATP lid, the apparent position of the open dwell level would have to be half its original value.

A closer analysis of the substrate-induced shift of the lower dwell levels is shown in Fig. 3c. For ATP, we find a substrate-dependent shift of the lower level (fraction of full closing) of ca. 0.2 as compared with the full closing distance. Apparently, the lid closes, but much less than full closing of one lid (0.5), let alone both lids (1.0). Two possible explanations outlined in Supplementary Fig. 10 could explain this result: (1) ATP binding occurs to a conformation with an only partially closed lid (pc) or (2) substrate binds to the open state with full affinity but drives AdK only weakly into a fc conformation resulting in a rapid equilibrium between $\mathrm{fc}$ and the open (o) conformation. Both of these explanations would support a conclusion that ATP binding does not strongly drive AdK into an fc ATP-lid state.

Analysis of the force dependence of the fraction of full closing (Fig. 3c) provides evidence for explanation (1) and hence the existence of a free-energy minimum in the AdK energy landscape at a pc position. We find that force only weakly affects the fraction of full closing as can be seen from the shallow slopes of the data points at all ATP concentrations. A fit to the data is consistent with a pc state (solid line fits) but not with an fc lid (dashed line; for a detailed model, see Methods). We cannot exclude transient population of the fc state; however, the major 
population will be the pc state. The concentration-dependent degree of ATP-induced lid closing extrapolated to zero force is shown in Fig. 3d (blue).

Similarly, in the presence of ADP, which binds to both lids, we also do not observe full closing of the enzyme. Even at the highest concentrations of ADP (1-10 mM) (see right trace in Fig. 3e), the ADP-bound level only approaches half of the fc state (Fig. 3d).

Phosphate linker length of inhibitor affects closing. How does the strong energetic drive towards the closed state of the enzyme in the presence of AP5A fit together with the population of a pc state and only a weak drive towards the $\mathrm{fc}$ state in the presence of substrates? A major difference between AP5A and two individual substrates is the rigid linkage between the adenosine moieties in AP5A, while individual substrates are free to move relative to each other. The rigid linkage in the inhibitor probably puts significant constraint on the conformational flexibility of AdK. Coupling of the relative distance between the substratebinding sites to the degree of lid closing could explain why the inhibitor locks the enzyme into the closed state: if only the closed conformation offers a binding site distance that matches the length of the phosphate linker, AP5A can only bind tightly to the closed state. We tested this hypothesis by measuring lid-closing distances with the inhibitors AP4A and AP6A, where the two adenosine moieties are linked with four or six phosphate groups, respectively (see Fig. 4a), thus altering their relative distance.

We found that AP4A induces full closing of AdK indistinguishable from AP5A (see Fig. 4b). In contrast, AP6A only closes to about $0.6 \mathrm{~nm}$, corresponding to a fraction of full closing of 0.4. One might argue that reduced closing due to AP6A as compared with the two other inhibitors is owed to the longer and bulkier phosphate chain that cannot be completely accommodated into an fc structure. However, the substratedependent closing and opening rates suggest a different explanation: we find that AP4A has a 100 -fold reduced closing rate (see Fig. 4c), which is consistent with a similarly reduced affinity for AP4A measured in bulk experiments ${ }^{21}$. For AP6A, we measure closing/binding rates that are identical to the ones of AP5A, showing that AP6A binds with the same affinity as AP5A despite its significantly reduced closing distance (see Fig. 4d). Zero-force opening rates for AP5A, AP4A and AP6A are largely identical (see Fig. 4e). It is noteworthy that the force dependence for opening of AP6A is weaker (smaller slope), consistent with the smaller conformational change (see Methods). Our results suggest that if the distance between the two adenosine moieties is larger, full lid closing for high affinity binding is not required. Hence, under these conditions, a pc state is already an optimal configuration.

Atomistic MD free-energy simulations of AdK bound to all three inhibitors support our conclusion and provide additional structural insight (Fig. $4 \mathrm{f}$ and for details, see Methods). In all cases, a significant free-energy minimum at a lid closing of $\sim 50 \%$ was observed, consistent with our interpretation that a pc state of the enzyme is a dominant feature of its energy landscape. For AP4A and AP5A binding with an added load of $15 \mathrm{pN}$, the fc state is of lower free energy than the pc state. In contrast, under the same conditions, for AP6A the pc state is lower in free energy than the $\mathrm{fc}$ state. Although a precise quantitative prediction of free energies cannot be expected given the known shortcomings of $\mathrm{MD}$ simulations, this result is at least in qualitative agreement with the experiment and in line with the experimentally observed dominant closing fractions in the inhibitor-bound states. Analysis of the simulation trajectories offers a structural explanation (see Supplementary Fig. 11). For the longer inhibitors, especially AP6A, a simultaneous binding of the two adenosine moieties to the corresponding lid sites is possible already at partially open AdK conformations. In case of the shorter AP4A inhibitor this stable binding mode is only possible at more closed lid configurations, which also offers an explanation for the reduced closing rate of AdK when AP4A is bound.

\section{Discussion}

The possibility of studying native-state conformational energies using mechanical single-molecule methods allows us to quantitatively determine the energetic drive of conformational changes of an enzyme induced by substrate binding. Even though structural studies have suggested firmly closed states, our results now provide an important enhancement of our understanding of AdKs conformational dynamics. The two apparently contradictory requirements of firm closing ensuring catalytic activity and flexibility to ensure rapid substrate/product exchange are met by (1) a strong drive towards a pc state with high substrate affinity still allowing for substrate exchange and (2) a much weaker drive into an $\mathrm{fc}$ and catalytically active structure that is only transiently visited. These results show that subnanometre force spectroscopy opens up new possibilities to directly study the energetic balance of enzyme conformations and help answering the question how these conformations couple to substrate binding.

How can our results be reconciled with reports from the literature that substrates induce full closing of $\mathrm{AdK}^{1,12,25}$ ? It is important to note that our measurements always occur under a certain amount of mechanical load. Therefore, a slight bias within the enzyme towards an fc state at zero load is well consistent with our findings. For example, Wolf-Watz et al. ${ }^{1}$ reported that under saturating substrate condition AdK rapidly fluctuates between closed and open conformations with $k_{\text {close }}=1,370 \mathrm{~s}^{-1}$ and $k_{\text {open }}=40 \mathrm{~s}^{-1}$, whereas Hanson et al. ${ }^{16}$ measure $k_{\text {close }}=400 \mathrm{~s}^{-1}$ and $k_{\mathrm{open}}=160 \mathrm{~s}^{-1}$ for E. coli AdK. Both these measurements indicate the open state is populated to between 3 and $30 \%$, consistent with our finding that despite the large substrate concentrations, the binding energy does not shift the equilibrium strongly to the closed state. Our data now explain where most of the substrate-binding free energy goes: it is used for populating a pc state. This may be important for the function of AdK as an enzyme that rapidly turns over a substrate and has to allow quick substrate exchange at the same time.

The results from the experiments with the three bisubstrate inhibitors AP4A, AP5A and AP6A can give a structural explanation for the occurrence of the pc state. Although AP4A and $\mathrm{AP} 5 \mathrm{~A}$ induce conformations where the adenosine moieties are close together, AP6A-bound AdK settles at a conformation where they are further apart (Fig. 4g right). The left branch of Fig. $4 \mathrm{~g}$ provides a picture of how the different states might blend into the catalytic cycle of AdK: when two unlinked substrate nucleotides bind, they will rapidly settle at an energetic minimum where the lids are half-closed with little energetic drive bringing them to the fc state. This will be the main substrate-bound state of the enzyme. In this state, catalysis cannot occur, because substrates are too far apart. Transiently, the $\mathrm{fc}$ state will then be visited in a process driven by thermal fluctuations. It is noteworthy that the fc state may even be a high-energy state and thus not energetically favoured (that is, no favourable energy bias) at least under the forces we apply. This $\mathrm{fc}$ state is essential for catalysis, because water molecules need to be excluded to ensure efficient phosphate transfer ${ }^{12,13}$. Even more than a single transition to the fc state may be necessary until catalysis occurs. After catalysis has occurred, the enzyme will switch back to the half open state and eventually release products. For rapid substrate turnover, it is important that the lids be not locked by favourable energy bias in an fc state but 
a
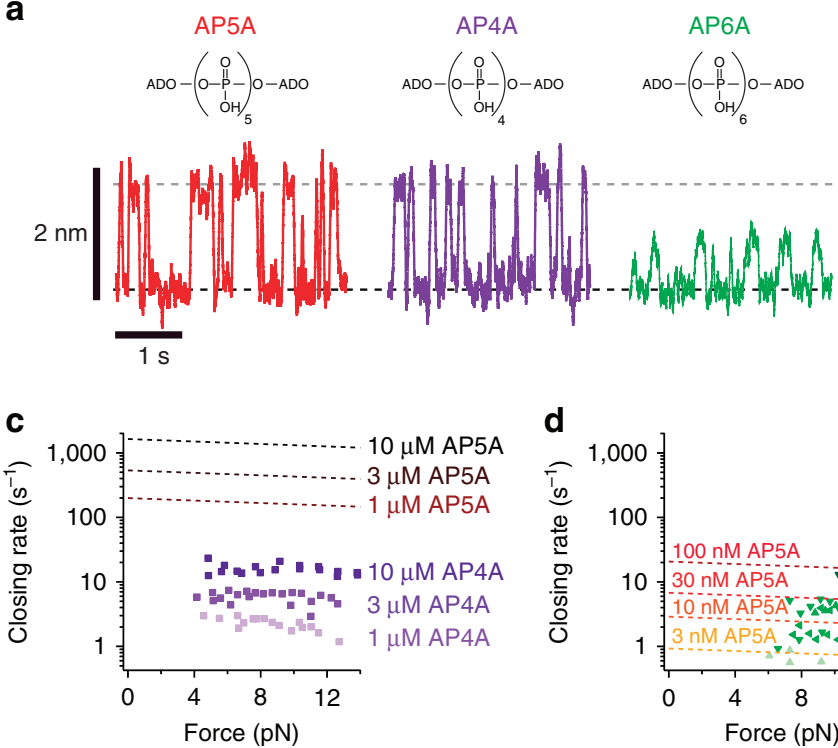

f
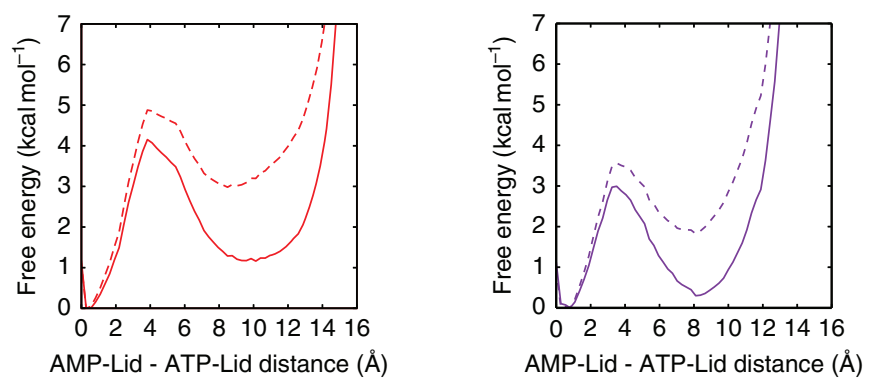

d

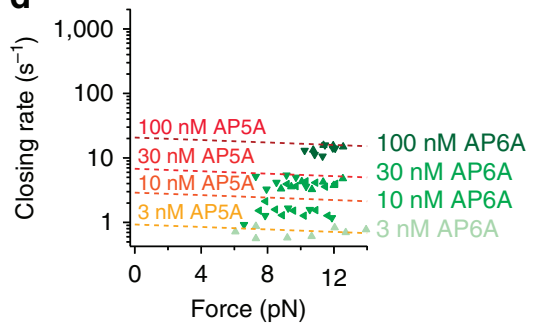

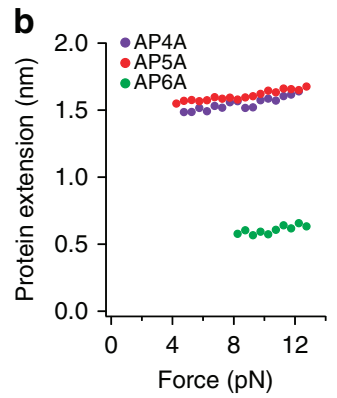

e

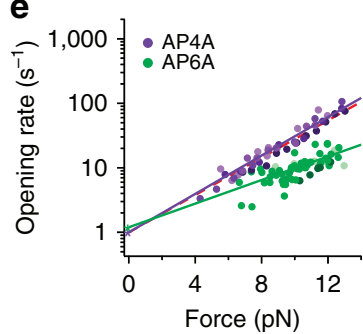

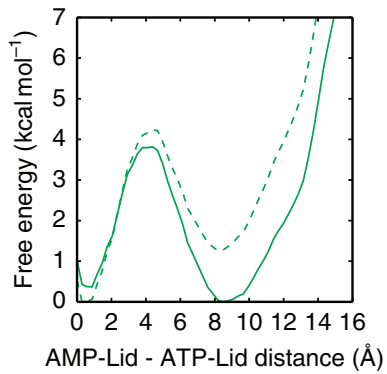

g

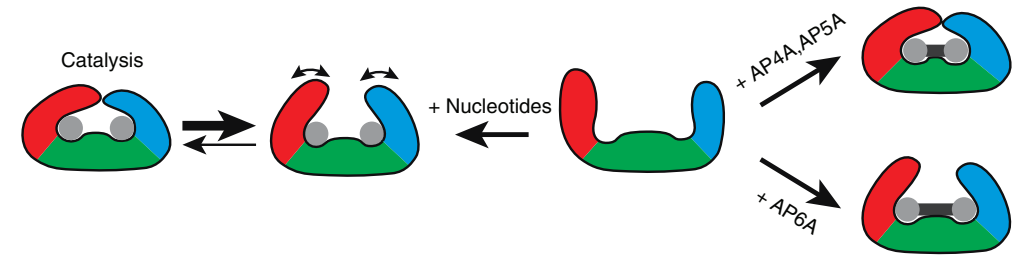

Figure 4 | Effect of the phosphate linker length between the adenosine moieties. (a) Equilibrium traces of the closing and opening of AdK in the presence of AP5A (left panel), AP4A (middle panel) and AP6A (right panel) at a force of $8 \mathrm{pN}$ for AP5A and AP4A, and $12 \mathrm{pN}$ for AP6A. The dashed lines represent the position of the fc (grey) and open (black) state of AdK. (b) Distance between closed and open state as a function of force in the presence of the different inhibitors AP5A (red), AP4A (purple) and AP6A (green). (c-d) Closing rate of AdK as a function of force for different AP4A (c) or AP6A (d) concentrations. It is noteworthy that the dashed lines are not fits to the AP4A and AP6A data, but fits to the rates obtained at the corresponding AP5A concentrations for comparison. (e) Opening rate of AdK as a function of force for different AP4A (purple) and AP6A (green) concentrations. Solid lines are fits to the closing rates extrapolated to zero force (asterisks). For comparison, a fit to the rates obtained at the corresponding AP5A concentrations is shown as a red dashed line. (f) Calculated free energy along the opening of AdK bound to AP5A (red), AP4A (purple) and AP6A (green) obtained from free-energy simulations without load (dashed lines) and with a subsequently added bias corresponding to an external load of $15 \mathrm{pN}$ (solid lines). The freeenergy curves are projections of free-energy landscapes (Supplementary Fig. 11) obtained from 2D H-REMD-US simulations in which the arrangements of AMP lid and ATP lid were controlled independently. (g) Model of the coupling between the conformational fluctuations and the binding of inhibitors or nucleotides to AdK. In the presence of nucleotides, a pc state is stably populated under force; however, AdK is still able to close fully especially in the absence of force. The binding of AP4A and AP5A induce the fc conformation of AdK, whereas the binding of AP6A stabilizes a pc state.

they open rapidly to allow product-substrate exchange. We think that the pc state ensures both rapid substrate exchange and highaffinity binding through establishing contacts between substrates, core domain and lids.

Why can we not see a kinetic time constant attributable to catalysis directly in our data? A turnover rate of $25 \mathrm{~s}^{-1}$ (that is, $40 \mathrm{~ms}$ ) in solution lies within the limit we should be able to resolve even in our filtered traces. However, as the fc state is only transiently populated, the lids will be only fully closed during a small fraction of those $40 \mathrm{~ms}$. In this case, we are not able to pick up a signal related to catalysis in the assay. In this context, it is important to note that we only have a readout for length changes but not for chemical changes.

Moreover, in our assay, we always apply loads around $10 \mathrm{pN}$, which, in turn will speed up opening kinetics and slow down closing kinetics. If force slows transitions to the fc state also, catalysis will be slowed and may be even suppressed, offering another explanation for why we cannot observe a signal associated with catalysis. A combination of mechanical and fluorescent single-molecule measurement may, in the future, give more insight into coupling between mechanical and chemical steps. 


\section{Methods}

Experimental procedures. The setup used was a custom-built, dual-beam, high-resolution optical trap setup as described previously ${ }^{26,27}$. Data were collected at $150 \mathrm{kHz}$ and then averaged to $30 \mathrm{kHz}$ before storage. Transitions between open and closed, and closed and open state were detected using Hidden Markov Model analysis on the unfiltered raw data of the difference signal of the two traps as described before ${ }^{28}$. The data at constant trap position shown throughout the study were low-pass filtered to $37.5 \mathrm{~Hz}$. All measurements were performed in $50 \mathrm{mM}$ Tris $\mathrm{pH} 7.5,200 \mathrm{mM} \mathrm{KCl}^{2} 26 \mathrm{U} \mathrm{ml}^{-1}$ glucose oxidase, $17,000 \mathrm{U} \mathrm{ml}^{-1}$ catalase, $0.65 \%$ glucose with varying concentrations of nucleotides, inhibitors, $\mathrm{MgCl}_{2}$ and EDTA. The $\mathrm{MgCl}_{2}$ or EDTA concentration was $2 \mathrm{mM}$ for nucleotide and inhibitor concentration below $1 \mathrm{mM}$, and twice the nucleotide concentration for nucleotide concentrations above $2 \mathrm{mM}$. All errors given are statistical errors (1 s.d.) derived from the Levenberg-Marquardt fit algorithm.

Protein expression and purification. The gene for AdK of A. aeolicus was synthesized by Mr Gene GmbH (Regensburg). The gene was cloned into pET11a using BamHI and NdeI restriction sites. Cysteine residues were inserted between positions 42 and 43, and 144 and 145 (wt-numbering) by QuikChange mutagenesis (Agilent). To observe conformational fluctuations of the ATP-lid only, a second mutant was produced with a cysteine at the amino terminus and a cysteine residue inserted between positions 144 and 145 (AdK N-144). The proteins were expressed in $E$. coli BL21(DE3) and purified by a Ni-NTA affinity column followed by gel filtration chromatography (HPLC system, Jasco Germany, GmbH) using YMC diol-120 columns (YMC Europe, GmbH) and Superdex S200 (10/300 GL, GE Healthcare). Attachment of oligonucleotides and purification optical trap assays were performed as described previously ${ }^{4,29}$, with an additional Ni-NTA affinity purification step for protein-oligonucleotide purification.

Protein sequences. In the following, the cysteines that are used for handle attachment are shown in bold, the sequence corresponding to AdK is shown in italics and the spacers are underlined.

AdK 42-144. MAAKGELSGMILVFLGPPGAGKGTQAKRLAKEKGFVHIS TGDILREA VQK $\overline{G C T P L G K K A K E Y M E R G E L V P D D L I I A L I E E V F P K H G N V I F D ~}$ GFPRTVKQAEALDEMLEKKGLKVDHVLLFEVPDEVVIERLSGRRINPET GEVYHVKYNPPPPGCVKVIQREDDKPEVIKKRLEVYREQTAPLIEYYKKKG ILRIIDASKPVE EVYRQVLEVIGDGNGTGSKALEHHHHHH.

AdK N-144. MACKGELSGMILVFLGPPGAGKGTQAKRLAKEKGFVHIST GDILREAVQKGTPLGKKAKEYMERGELVPDDLIIALIEEVFPKHGNVI FDGFPRTVKQAEALDEMLEKKGLKVDHVLLFEVPDEVVIERLSGRRINPE TGEVYHVKY NPPPPGCVKVIQREDDKPEVIKKRLEVYREQTAPLIEYYKK KGILRIIDASKPVEEVYRQVL EVIGDGNGTGSKALEHHHHHH.

Enzymatic activity of AdK. The enzymatic activity of the AdK mutant was tested by a coupled spectrophotometric assay ${ }^{30}$ (Supplementary Figs 12 and 13). The resulting Michaelis-Menten constant $K_{\mathrm{m}}(41 \pm 11 \mu \mathrm{M}$ for ATP, $\sim 10 \mu \mathrm{M}$ for $\mathrm{AMP}$ and $360 \pm 80 \mu \mathrm{M}$ for $\mathrm{Mg}-\mathrm{ADP} / \mathrm{ADP})$ and the catalytic rate $k_{\text {cat }}\left(25 \pm 7 \mathrm{~s}^{-1}\right)$ are in good agreement with the values of the wild-type protein reported by Wolf-Watz et al. ${ }^{1}$

DNA elasticity correction. As our measurements occur at non-constant force, comparing the conformational changes of AdK under different experimental conditions requires corrections for trap and DNA compliances. We therefore corrected all traces obtained at constant trap distances for the non-Hookean elastic behaviour of the double-stranded DNA linker. Briefly, a force-extension curve of a DNA-protein construct was measured. Data, force versus time, were acquired, and from known distance and trap stiffness, force-extension curves were calculated. Next, the data were fitted by worm-like chain (WLC) model to account for the DNA tether $(\sim 360 \mathrm{~nm})$. From the now known WLC model parameters, the extension of the DNA handles can be calculated for each data point and subsequently subtracted. This 'constant-force' transformation removes variations in the DNA linker elasticity and trap stiffness, and allows for a better comparison of the conformational changes of AdK between different experiments. All data shown are subjected to this procedure.

Extrapolation of closing and opening rates. For determining the opening and closing rates at zero force $k_{0, i}$ and distances to the transition state $\Delta x$, the force-dependent opening and closing rates $k_{i}(F)$ were fitted with a Bell model ${ }^{31}$ :

$$
k_{i}(F)=k_{0, i} \exp \left(\frac{F \Delta x_{i}}{k_{\mathrm{B}} T}\right),
$$

where $k_{\mathrm{B}}$ is the Boltzmann constant and $T$ is the absolute temperature. The distance to the transition state is a measure for the force dependence of the respective rate. A small $\Delta x$ results in a small force dependence of the corresponding rate, whereas a large $\Delta x$ causes the opening or closing rate to be strongly dependent on force. This behaviour can be observed for the conformational change in the presence of AP5A and AP6A. Both feature a similar zero-force opening rate; however, the force dependence of the AP5A opening rate is much bigger, as the size of the conformational change and with it the distance to the transition state is larger.

Bell model fits were performed as global fits to the closing rate for the individual inhibitors, assuming the same distance to the transitions state $\Delta x$ for all concentrations and individual zero force closing rates $k_{0, \text { close. }}$ The Bell model fits to the opening rate were performed as global fits for the individual inhibitors, assuming the same distance to the transitions state $\Delta x$ and the same zero force opening rate $k_{0, \text { open }}$.

Equilibrium free energies. Equilibrium free energies of the conformational fluctuations of AdK in the presence of inhibitors and in the competition assay were calculated as described previously ${ }^{4}$

Affinities from competition assay. The affinity of the substrates to AdK was determined as follows. First, the binding free energy of AP5A at various AP5A concentration, $\Delta G^{0}$, was determined in the absence of nucleotides as described before. Second, the decrease in binding free energy due to nucleotide binding in the AP5A competition assay was determined by comparison with the AP5A-only conditions. The nucleotide concentration-dependent decrease in binding free energy can be described by

$$
-\frac{\Delta G^{0}}{k_{\mathrm{B}} T}=-\ln \left(1+\frac{[L]}{K_{\mathrm{D}}}\right),
$$

where $[L]$ is the nucleotide concentration and $K_{\mathrm{D}}$ the dissociation constant of the nucleotides to $\mathrm{AdK}^{32}$.

ATP lid unfolding and free energy. Stretching AdK at positions 42 and 144 shows a continuous hump-like equilibrium transition around a force of $17 \mathrm{pN}$ in force extension traces (see Supplementary Fig. 6). These fast unfolding and folding transitions can be observed between 13 and $20 \mathrm{pN}$. To calculate the free energy of this transition, we integrated the force distance curves. The free energy of the whole system, including the stretching of the DNA linker, is given by the area under the force distance curve of a constant velocity experiment (for a clearer representation, Supplementary Fig. 8a shows the corresponding curves as force extension traces). To calculate the energy of the system in the unfolded state, a serial combination of DNA and polypeptide elasticity is fit to the data and the energy of the system in the unfolded state is given by the area under the fit. To reduce the influence of noise at low forces, a WLC model is also fit to the folded state. The energy of the whole system is calculated by the sum of the area under the WLC fit to the folded state up to a force of $10 \mathrm{pN}$ and the area under the actual curve (orange) for forces above $10 \mathrm{pN}$. By subtracting the free energy of the system in the unfolded state (grey area) from the free energy of the whole system, we obtain the free energy of the folding/ unfolding equilibrium transition (blue area).

The free energy of the folding/unfolding equilibrium transition in apo conditions is $(11.7 \pm 0.7) k_{\mathrm{B}} T$. The increase in free energy of this transition in presence of the nucleotides Mg-ADP and Mg-ATP is shown in Supplementary Fig. 8b.

The free energy in the presence of nucleotides can be described similar to the competition assay as

$$
-\frac{\Delta G_{0}}{k_{\mathrm{B}} T}=-\frac{\Delta G_{0}^{L=0}}{k_{\mathrm{B}} T}-\ln \left(1+\frac{[L]}{K_{\mathrm{D}}}\right),
$$

where $[L]$ is the nucleotide concentration, $-\frac{\Delta G_{0}^{L=0}}{k_{\mathrm{B}} T}$ is the free energy in apo conditions and $K_{\mathrm{D}}$ the dissociation constant of the nucleotides to AdK.

Nucleotide-induced conformational fluctuations. To determine the size of the conformational change induced by the nucleotides, the measured size of the AP5Ainduced conformational fluctuations in the competition experiment is compared with the one at AP5A-only conditions. The fraction of closing induced by the nucleotides in the competition experiment is given by the difference between the size of the conformational change at AP5A-only conditions compared with the one in the presence of nucleotides in the competition experiment and normalized by the length of the conformational change in presence of AP5A only:

$$
\operatorname{Frac}(F)=\frac{L_{\mathrm{AP} 5 \text { Aonly }}(F)-L_{\mathrm{NuclComp}}(F)}{L_{\mathrm{AP} 5 \text { Aonly }}(F)},
$$

where $L_{\mathrm{AP} 5 \mathrm{Aonly}}(F)$ is the size of the full conformational change for AP5A-only experiments (in $\mathrm{nm}$ ) and $L_{\mathrm{NuclComp}}(F)$ is the size of the conformational change in the nucleotide competition experiment. The system can be described by the probability to find the enzyme in either the open or closed state. The forcedependent probability to find the enzyme in the closed state is given by

$$
P_{\text {closed }}(F)=\frac{k_{\text {close }}(F)}{k_{\text {close }}(F)+k_{\text {open }}(F)}=\frac{1}{1+\frac{k_{\text {open }}(F)}{k_{\text {close }}(F)}},
$$

where $k_{\text {close }}(F)$ and $k_{\text {open }}(F)$ are the force-dependent closing and opening rates at a specific nucleotide concentration, respectively. Assuming the Bell model, with a 
zero force rate $k_{0, i}$ and a distance to the transition state $\Delta x_{i}$, the force dependence of the rates is given by equation (1).

With equation (1), the force dependence of the probability to find $\mathrm{AdK}$ in the closed state can be rewritten as:

$$
P_{\text {closed }}(F)=\frac{1}{1+\frac{k_{0, \text { open }}}{k_{0, \text { close }}} \exp \left(\frac{F \Delta x}{k_{\mathrm{B}} T}\right)} .
$$

Here, $k_{0, \text { open }}$ and $k_{0 \text {,close }}$ are the zero-force opening and closing rates, respectively, and $\Delta x$ is the size of the conformational change that AdK undergoes in the presence of nucleotides. To calculate the fraction of closing, equation (6) has to be extended by $A=\frac{\Delta x}{1.72 \mathrm{~mm}}$

$$
\operatorname{Frac}(F)=\frac{A}{1+\frac{k_{0, \text { open }}}{k_{0, \text { close }}} \exp \left(\frac{F \Delta x}{k_{\mathrm{B}} T}\right)} .
$$

A global fit of this model to the force-dependent fraction of closing at all ATP concentration was performed. One $\Delta x$ for all ATP concentrations was used and an ATP concentration-dependent ratio $\frac{k_{0,0 p e n}}{k_{0, \text { dose }}}$ was used as well. The global fit to the data yields the size of the conformational change induced by ATP of $\Delta x=0.5 \mathrm{~nm}$. A similar global fit to the force-dependent fraction of closing shown in Supplementary Fig. 9 for different Mg-ADP concentrations yields the size of the conformational change induced by Mg-ADP of $\Delta x=0.7 \mathrm{~nm}$. From the acquired fit parameters, the fraction of full closing at zero force can be determined (Fig. 3d).

To describe the concentration-dependent closing of AdK in presence of a ligand, the probability to find the enzyme in the closed conformation in the presence of a ligand is calculated

$$
P_{\text {closed }}=\frac{[B C]+[U C]}{[B C]+[U C]+[B O]+[U O]},
$$

where $[\mathrm{BC}]$ and $[\mathrm{UC}]$ are the concentrations of ligand-bound and ligand-unbound closed state, respectively, and $[\mathrm{BO}]$ and $[\mathrm{UO}]$ are the concentrations of ligandbound and ligand-unbound open state, respectively. The dissociation constants for the open $K_{\text {open }}$ and closed state $K_{\text {close }}$ are defined by

$$
K_{\mathrm{open}}=\frac{[U O] * c}{[B O]},
$$

and

$$
K_{\text {close }}=\frac{[U O] * c}{[B C]} .
$$

Single-molecule fluorescence experiments by Henzler-Wildman et al. ${ }^{18}$ have shown that the equilibrium between open and closed state in the absence of nucleotides lies on the open side (opening:closing rate, $6,500 \mathrm{~s}^{-1}: 2,000 \mathrm{~s}^{-1}$ ). Assuming a similar $\Delta x$ for full closing as in the AP5A case, force will shift the equilibrium further towards the open side. At our measurement forces, the concentration [UC] will be negligible compared with the other concentrations. Together with equations (9) and (10), equation (8) can be simplified to:

$$
P_{\text {closed }}(c)=\frac{1}{1+K_{\text {close }}\left(\frac{1}{c}+\frac{1}{K_{\text {open }}}\right)}
$$

As the size of the conformational change caused by the nucleotides is known from the fits of the force-dependent closing data, the concentration-dependent fraction of closing can be written as:

$$
\operatorname{Frac}(c)=\frac{A}{1+K_{\text {close }}\left(\frac{1}{c}+\frac{1}{K_{\text {open }}}\right)} .
$$

MD simulation. All-atom MD simulations were performed on AdK bound to each of the three inhibitors AP4A, AP5A and AP6A. Two-dimensional (2D) potentials of mean force (PMFs) along the opening of the two flexible lid domains were calculated by means of Hamiltonian-replica exchange umbrella sampling (H-REMD-US) simulations. The resulting 2D free-energy landscapes were projected on a one-dimensional coordinate, resembling the experimental lid-to-lid distance. A free-energy bias corresponding to a force of $15 \mathrm{pN}$ was subsequently added to the free-energy projections, to enable direct comparison with the experiments.

Starting structures. The crystal structure of A. aeolicus AdK in complex with AP5A was taken from PDB:2RGX ${ }^{18}$. Structures of AP4A and AP6A were built manually and optimized with respect to their internal energy using Antechamber as part of Amber14 (ref. 33). Starting structures of AdK in complex with AP4A and AP6A were constructed by aligning ${ }^{34}$ these inhibitors over AP5A in 2RGX and subsequently removing AP5A (followed by energy minimization). Using the Leap module of Amber14, a periodic octahedral box with edge length $\sim 28 \AA$ was constructed around each protein-inhibitor complex and filled with $\sim 9,000$ water molecules. Potassium ions were added to neutralize the overall charge of the systems.
Simulation setup. All simulations were carried out using Amber14 (ref. 33). The protein was described by the Amber ff14SB force field ${ }^{35}$ and the water molecules by the TIP3P model ${ }^{36}$. The AP4A, AP5A and AP6A inhibitors were parametrized with Antechamber and the GAFF ${ }^{37}$ force field. Specific parameters were used for the potassium ions ${ }^{38}$. The short-range cutoff radius was $9 \AA$. Long-range interactions were accounted for by the particle mesh Ewald method (Amber14 default parameters). Hydrogen bonds were constrained via the SHAKE $^{39}$ algorithm. The integration time step was $2 \mathrm{fs}$. For temperature and pressure coupling, Berendsen thermostat and barostat ${ }^{40}$ were used with a coupling time of 1 ps.

Equilibration. The starting structures were minimized with respect to their internal energy using the steepest decent method for 10,000 steps. Several equilibration simulations of $20 \mathrm{ps}$ were performed in the NPT ensemble at a pressure of 1 bar. Initially, the temperature was successively raised to 100,200 and $298 \mathrm{~K}$, while harmonic positional restraints of a force of $50.0 \mathrm{kcal} \mathrm{mol}^{-1} \AA^{-2}$ were applied to protein and inhibitor atoms with respect to the starting structures. In five additional simulations, the restraining potential force was successively reduced to 24.0 , $12.0,6.0,2.0$ and $0.2 \mathrm{kcal} \mathrm{mol}^{-1} \AA^{-2}$. A final equilibration step of $40 \mathrm{ps}$ was performed without restraints. The total equilibration time of each system was $2 \mathrm{~ns}$.

2D H-REMD-US simulations. The US simulations were performed in the NVT ensemble at $298 \mathrm{~K}$. The reaction coordinates used for the $2 \mathrm{D} \mathrm{US}^{41}$ simulations were defined as the centre of mass distances between the $\mathrm{C}_{\alpha}$-atoms of residues 33-58 and 114-123, 154-170 (AMP-lid opening) and 125-148, and 1-28 and 75-82 (ATP-lid opening), respectively. Harmonic umbrella potentials were placed along the AMP-lid coordinate between 18.0 and $30.1 \AA$, and along the ATP-lid coordinate between 29.0 and $31.1 \AA$ with an intermediate spacing of $1.1 \AA$. To obtain fast equilibration of the US simulation setup, two different starting configurations for each umbrella window were generated. The equilibrated structures were driven along the ATP-lid coordinate and then along the AMP-lid coordinate by applying the umbrella potentials one after another with a force constant of $4 \mathrm{kcal} \mathrm{mol}^{-1} \AA^{-2}$ for 100 ps. The process was then repeated in the reverse order, starting from the obtained open AdK conformation. Only during this initial process, the inhibitor atoms were restrained to their initial positions with a force of $0.025 \mathrm{kcal} \mathrm{mol}^{-1} \AA^{-2}$, to prevent dissociation due to the fast initial opening motion. In this way, starting configurations generated on the basis of a completely open and a completely closed structure were provided for each umbrella window position. Subsequently, two strands of 2D US simulations with a harmonic force constant of $2 \mathrm{kcal} \mathrm{mol}^{-1} \AA^{-2}$ were run in parallel, each based on one of the previously obtained sets of starting configurations. Via Hamiltonian replica exchanges ${ }^{42}$, neighbouring windows within and between the two strands were allowed to swap configurations. This enables rapid diffusion of the configurations along the reaction coordinate plane and between the strands as well. The exchange scheme was realized by performing $4 \mathrm{D}$ H-REMD-US with adequately modified exchange groups. As starting configurations originating from two configurational extremes are provided, which are allowed to rapidly exchange towards an adequate umbrella window position, equilibration is drastically sped up in comparison with a setup based on only one starting configuration per window. For the actual sampling, the umbrella windows at the positions between 29.0 and $30.1 \AA, 18.0$ and $21.3 \AA$ (top left region), and between 19.0 and $22.3 \AA$ (bottom right region, see Supplementary Fig. 11) of the reaction coordinate plane were omitted, to obtain an advantageous number of 256 parallel simulations. Replica exchanges were attempted every 1 ps with rates of successful exchanges between 0.06 and 0.29 . Each umbrella window was simulated for $6 \mathrm{~ns}$ ( $12 \mathrm{~ns}$ per umbrella window position), totalling up to $>1.5 \mu \mathrm{s}$ of sampling time for each system. Reaction coordinate values were saved every $0.1 \mathrm{ps}$. The $2 \mathrm{D}$ PMFs were calculated from the sampling data using the Weighted Histogram Analysis Method ${ }^{43,44}$. The first $2 \mathrm{~ns}$ of sampling time were omitted in the PMF calculations, to ensure adequate equilibration of the umbrella windows.

Free-energy projection and additional external force. To enable a direct comparison of the MD results with the experiments, the 2D free -energy landscapes obtained from the simulations (Supplementary Fig. 11) were projected on a coordinate roughly resembling the lid-to-lid distance as follows:

$$
d_{\text {lid-lid }}\left(d_{\text {AMP-lid }}, d_{\text {ATP-lid }}\right)=\sqrt{\left(d_{\text {AMP-lid }}-d_{0, \text { AMP-lid }}\right)^{2}+\left(d_{\text {ATP-lid }}-d_{0, \text { ATP-lid }}\right)^{2}} .
$$

Here, $d_{\mathrm{AMP}-\text { lid }}$ and $d_{\mathrm{ATP}-\text { lid }}$ are the lid opening coordinates used in the H-REMDUS simulations, and $d_{0, \mathrm{AMP}-\text { lid }}$ and $d_{0, \mathrm{ATP} \text {-lid }}$ are the locations of the global minima in the free-energy landscapes. We hereby assumed that the lid-domain motions are mainly orthogonal. A bias corresponding to the load exerted during the experiments was subsequently added via

$$
\operatorname{PMF}_{\text {biased }}\left(d_{\text {lid-lid }}\right)=\operatorname{PMF}\left(d_{\text {lid-lid }}\right)-F_{\text {external }} \times d_{\text {lid-lid }}-\operatorname{PMF}_{0, \text { biased }} .
$$

As an external force $F_{\text {external }} 15 \mathrm{pN}$ was used, representative for the loads applied during the experiments. Finally, the resulting new minima of the biased PMFs were shifted to zero. 


\section{References}

1. Wolf-Watz, M. et al. Linkage between dynamics and catalysis in a thermophilic-mesophilic enzyme pair. Nat. Struct. Mol. Biol. 11, 945-949 (2004).

2. Pisliakov, A. V., Cao, J., Kamerlin, S. C. \& Warshel, A. Enzyme millisecond conformational dynamics do not catalyze the chemical step. Proc. Natl Acad. Sci. USA 106, 17359-17364 (2009).

3. Itoh, H. et al. Mechanically driven ATP synthesis by F1-ATPase. Nature 427, 465-468 (2004).

4. Stigler, J., Ziegler, F., Gieseke, A., Gebhardt, J. C. M. \& Rief, M. The complex folding network of single calmodulin molecules. Science 334, 512-516 (2011).

5. Svoboda, K., Schmidt, C. F., Schnapp, B. J. \& Block, S. M. Direct observation of kinesin stepping by optical trapping interferometry. Nature 365, 721-727 (1993)

6. Wen, J.-D. et al. Following translation by single ribosomes one codon at a time. Nature 452, 598-603 (2008).

7. Rief, M., Gautel, M., Oesterhelt, F., Fernandez, J. M. \& Gaub, H. E. Reversible unfolding of individual titin immunoglobulin domains by AFM. Science 276, 1109-1112 (1997)

8. Woodside, M. T. et al. Direct measurement of the full, sequence-dependent folding landscape of a nucleic acid. Science 314, 1001-1004 (2006).

9. Liphardt, J., Onoa, B., Smith, S. B., Tinoco, Jr I. \& Bustamante, C. Reversible unfolding of single RNA molecules by mechanical force. Science 292, 733-737 (2001).

10. Bae, E. \& Phillips, G. N. Roles of static and dynamic domains in stability and catalysis of adenylate kinase. Proc. Natl Acad. Sci. USA 103, 2132-2137 (2006).

11. Rundqvist, L. et al. Noncooperative folding of subdomains in adenylate kinase. Biochemistry 48, 1911-1927 (2009).

12. Kerns, S. J. et al. The energy landscape of adenylate kinase during catalysis. Nat. Struct. Mol. Biol. 22, 124-131 (2015).

13. Berry, M. B. et al. The closed conformation of a highly flexible protein: the structure of E. coli adenylate kinase with bound AMP and AMPPNP. Proteins 19, 183-198 (1994).

14. Müller, C. W. \& Schulz, G. E. Structure of the complex between adenylate kinase from Escherichia coli and the inhibitor Ap5A refined at 1.9 A resolution. A model for a catalytic transition state. J. Mol. Biol. 224, 159-177 (1992).

15. Rhoads, D. G. \& Lowenstein, J. M. Initial velocity and equilibrium kinetics of myokinase. J. Biol. Chem. 243, 3963-3972 (1968).

16. Hanson, J. A. et al. Illuminating the mechanistic roles of enzyme conformational dynamics. Proc. Natl Acad. Sci. USA 104, 18055-18060 (2007).

17. Arora, K. \& Brooks, C. L. Large-scale allosteric conformational transitions of adenylate kinase appear to involve a population-shift mechanism. Proc. Natl Acad. Sci. USA 104, 18496-18501 (2007).

18. Henzler-Wildman, K. A. et al. Intrinsic motions along an enzymatic reaction trajectory. Nature 450, 838-844 (2007).

19. Whitford, P. C., Gosavi, S. \& Onuchic, J. N. Conformational transitions in adenylate kinase. Allosteric communication reduces misligation. J. Biol. Chem. 283, 2042-2048 (2008).

20. Lienhard, G. E. \& Secemski, I. I. P 1 ,P 5 -Di(adenosine-5')pentaphosphate, a potent multisubstrate inhibitor of adenylate kinase. J. Biol. Chem. 248, 1121-1123 (1973).

21. Reinstein, J. et al. Fluorescence and NMR investigations on the ligand binding properties of adenylate kinases. Biochemistry 29, 7440-7450 (1990).

22. Elms, P. J., Chodera, J. D., Bustamante, C. J. \& Marqusee, S. Limitations of constant-force-feedback experiments. Biophys. J. 103, 1490-1499 (2012).

23. Kalbitzer, H. R., Marquetant, R., Rösch, P. \& Schirmer, R. H. The structural isomerisation of human-muscle adenylate kinase as studied by $1 \mathrm{H}$-nuclear magnetic resonance. Eur. J. Biochem. 126, 531-536 (1982).

24. Schrank, T. P., Bolen, D. W. \& Hilser, V. J. Rational modulation of conformational fluctuations in adenylate kinase reveals a local unfolding mechanism for allostery and functional adaptation in proteins. Proc. Natl Acad. Sci. USA 106, 16984-16989 (2009).

25. Adén, J. \& Wolf-Watz, M. NMR identification of transient complexes critical to adenylate kinase catalysis. J. Am. Chem. Soc. 129, 14003-14012 (2007).

26. von Hansen, Y., Mehlich, A., Pelz, B., Rief, M. \& Netz, R. R. Auto- and crosspower spectral analysis of dual trap optical tweezer experiments using Bayesian inference. Rev. Sci. Instrum. 83, 095116 (2012).

27. Zoldák, G., Stigler, J., Pelz, B., Li, H. \& Rief, M. Ultrafast folding kinetics and cooperativity of villin headpiece in single-molecule force spectroscopy. Proc. Natl Acad. Sci. USA 110, 18156-18161 (2013).

28. Stigler, J. \& Rief, M. Hidden markov analysis of trajectories in single-molecule experiments and the effects of missed events. Chemphyschem 13,1079-1086 (2012).
29. Cecconi, C., Shank, E. a., Bustamante, C. \& Marqusee, S. Direct observation of the three-state folding of a single protein molecule. Science 309, 2057-2060 (2005).

30. Saint Girons, I. et al. Structural and catalytic characteristics of Escherichia coli adenylate kinase. J. Biol. Chem. 262, 622-629 (1987).

31. Bell, G. I. Models for the specific adhesion of cells to cells. Science 200, 618-627 (1978).

32. Bodenreider, C. \& Kiefhaber, T. Interpretation of protein folding psi values. J. Mol. Biol. 351, 393-401 (2005).

33. Case, D. A. et al. AMBER 14 (Univ. California, 2014).

34. The PyMOL Molecular Graphics System, Version 1.7.4 (Schrodinger, LLC (2010).

35. Hornak, V. et al. Comparison of multiple Amber force fields and development of improved protein backbone parameters. Proteins 65, 712-725 (2006).

36. William, L. J., Jayaraman, C., Jeffry, D. M., Roger, W. I. \& Michael, L. K. Comparison of simple potential functions for simulating liquid water. J. Chem. Phys. 79, 926-935 (1983).

37. Wang, J., Wolf, R. M., Caldwell, J. W., Kollman, P. A. \& Case, D. A. Development and testing of a general Amber force field. J. Comput. Chem. 25, 1157-1174 (2004).

38. Joung, I. S. \& Cheatham, T. E. Determination of alkali and halide monovalent ion parameters for use in explicitly solvated biomolecular simulations. J. Phys Chem. B 112, 9020-9041 (2008).

39. Ryckaert, J. P., Ciccotti, G. \& Berendsen, H. J. C. Numerical-integration of Cartesian equations of motion of a system with constraints - moleculardynamics of N-alkanes. J. Comput. Phys. 23, 327-341 (1977).

40. Berendsen, H. J. C., Postma, J. P. M., van Gunsteren, W. F., DiNola, A. \& Haak, J. R. Molecular dynamics with coupling to an external bath. J. Chem. Phys. 81, 3684-3690 (1984).

41. Torrie, G. M. \& Valleau, J. P. Nonphysical sampling distributions in Monte Carlo free-energy estimation: Umbrella sampling. J. Comput. Phys. 23, 187-199 (1977).

42. Fukunishi, H., Watanabe, O. \& Takada, S. On the Hamiltonian replica exchange method for efficient sampling of biomolecular systems: application to protein structure prediction. J. Chem. Phys. 116, 9058-9067 (2002).

43. Kumar, S., Rosenberg, J. M., Bouzida, D., Swendsen, R. H. \& Kollman, P. A. The weighted histogram analysis method for free-energy calculations on biomolecules. I. The method. J. Comput. Chem. 13, 1011-1021 (1992).

44. Grossfield, A. WHAM: the weighted histogram analysis method 2.0.7. http://membrane.urmc.rochester.edu/content/wham (2013).

45. Okazaki, K.-I. \& Takada, S. Dynamic energy landscape view of coupled binding and protein conformational change: induced-fit versus population-shift mechanisms. Proc. Natl Acad. Sci. USA 105, 11182-11187 (2008).

\section{Acknowledgements}

This work was supported by an SFB 1035 grant of Deutsche Forschungsgemeinschaft to M.R. (A05) and M.Z. (B02).

\section{Author contributions}

B.P. performed experiments. B.P., G.Z. and M.R. designed research, analysed and discussed data, and provided analytical tools and materials. F.Z. and M.Z. performed and analysed MD simulations. B.P., G.Z., F.Z., M.Z. and M.R. prepared figures, wrote and edited the manuscript.

\section{Additional information}

Supplementary Information accompanies this paper at http://www.nature.com/ naturecommunications

Competing financial interests: The authors declare no competing financial interests

Reprints and permission information is available online at http://npg.nature.com/ reprintsandpermissions/

How to cite this article: Pelz, B. et al. Subnanometre enzyme mechanics probed by single-molecule force spectroscopy. Nat. Commun. 7:10848 doi: 10.1038/ncomms10848 (2016).

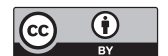

This work is licensed under a Creative Commons Attribution 4.0 International License. The images or other third party material in this article are included in the article's Creative Commons license, unless indicated otherwise in the credit line; if the material is not included under the Creative Commons license, users will need to obtain permission from the license holder to reproduce the material. To view a copy of this license, visit http://creativecommons.org/licenses/by/4.0/ 